\title{
VIII. Wanderung durch die Sammlung von Siegelabgussen im k. allg. Reichsarchiv zu Manchen. Von
}

K. Primbs, k. Reichsarchivrath.

(Fortsetzung.)

Als im rerflossenen Jahre diese Wanderung angetreten ward, geschah es in der Voraussetzung, dass so Manchem das Ergebniss derselben erwünscht sein werde, da gerade in der Abtheilung Adel in reicherer Zahl sich Siegel finden dürften, die nur einem kleinen Kreise von Fachmănnern bekannt sind. Vermogen doch nur wenige sich den Aufwand an Zeit and Geld zu gestatten, um selbst in den Archiven heraldisch-sphragistischen Forschungen sich hinzugeben.

Die von verschiedenen Seiten dem Unternehmen gezollte Anerkennung, wie der mehrfach zum Ausdruck gelangte Wunsch, dass diese Wanderung fortgesetzt werden möge, liess die folgenden Zeilen entstehen. Auch diesmal wurden Siegel des Adels gewählt, da immer, wenn auch jetzt in der Zeit schon etwas weiter herabgegriffen werden musste, noch hinreichend interessantes Material vorhanden.

Da nach dem Abdrucke des ersten Theiles der Beschreibung hie und da noch Einzelnes zu ergänzen, auch zu berichtigen räthlich erschien, wurde hier am Schlusse eine kurze Mittheilung angehängt.

\section{Abtheilung.}

Adel.

Abersfeld v., Frizo. 1335. Schrigbalke. F.

Ach dorf v., Heinrich. 1336. Angel $B$.

A derzhausen v., Ott. 1378. Schragbalke. B.

A esenheim v., Alram. 1332. Zwei Balken. B.

A eusenhofen (Eisenhofen) v., Cath. 1337. Im Siegelfeld ein Vogel. B.

Aff al te rberger (Opfolterberg), Hilprant. 1332. Drei A epfel auf dem Stulp des mit 7 Federn besteckten Hutes. Oes.

Aindorfer, Con. 1351. Abgekehrte Pferdebusten. B. 
A isterheimer, Erhard. 1335. Veh. unt. Haupt. B.

Alburger, Con. 1318. Anf dem Helm Federn besteckt. Bockshorn. B.

A m rang v., Niklas. 1303. Aufrechte Zange.

Angel berger, Sigmund. 1399. Gestiurste Angel. B.

A rnbeck v., Seif. 1322. Spitze. B.

A rnsberg v., Hadebrand. 120̄3. $\nabla$ sechsmal gestückt. von 2 Stern. beseit. Schrigbalke. F.

A scha u v., Ortlieb. 1333. Gekürte absteig. Spitze. Ortlieb. 1303. Dreiberg. B. A uer, Hans. 1334. Lowenkopf. B.

A uer v. Stefning, Dietrich. 1365. Drei Rosen auf Schrägbalken. Sind die Regensburger Auer.

Panholz, Rup. 1330. Drei Keile anf Schrigbalken. B.

Pappenheim v., Friedrich. 1360. Gekrönter Mohrenkopf. Oest.

Pas s a n v., Ulrich. 1304. Geschrigt, unten geralut.

- Christian. $\nabla$ Wappen wie Ulrich.

- Friedrich. 1272. Zackenbalke.

- Leutwein. 1304. Zugekehrte Pferdewedel wie das Wappen des Gameret v. Sarching in Regensburg.

Paner v. Allersburg, Frledrich. 1341. Drei aufsteigende Flammen. B.

Bebenburg v., Lapold. 0 zweitharmige Burg. Milt. Arch. F.

- Rudolph. 1337. Gleiches Wappen.

Peis do rf v., Ott. 1323. Steig. Ochse. B.

Belriet v., Theodor. 1335. Im Siegelfeld behut. Mannsbuste. $F$.

Benesch, Diet. 1305. An ein. Ring befest. gegen die Ecken gekehrt. Federn. Oes.

Bercheim v., Marchard. 1331. Helm: Rad auf Kissen. Oest.

Perger, Ulich. 1322. Halbes gekröntes Einhorn. In der Siegelleg: U. v. Chnollenperg. B.

- Berlepsch v., Arnold. 1350. Drei Sparren. Hess.

Bersd orf v., Reimpert. 1286. „Camerar. Aust.“ Auf Fels steh. Hirsch. Oest.

Peurn v., Walth Burggraf v. Schwandorf. Aufsteig. Blatt. Oest.

Pfalzpeunt v., Herm. 1332. 2. 1. Sterne. B.

Pfefferbalch, Con. 1342. Viermal geschrigt. $B$.

Pfers d o rf v., Gerlach. 1334. $\nabla$ steh. gezaumt. Ross. F.

Pybarn v., Bertold. 1304. Adler. Oest.

Bibera v., Hermann. Vorbrech. Bracke. Milt. Arch.

Piberach $\nabla .$, Con. 1349. Bieber.

- Heinrich. 1349. Steig. Bieber.

Pillnacher, Con. 1338. Geschränkte Hiammer anf gequ. Schild. B.

Planke n peck v., Raffald. 1333. Zackenbalke. B.

Pless, Otto. 1330. Von drei Sternen beseit. Sparre. B.

Plesse v., Gotschalk. Herr v. Maneranker. - Fenereisen? - 1356. Th.

Plico, Rainold. 1333. Fisch vor ferderbesteckt. Stander. Boe.

Bockesberg v., Ulrich. 1315. Steh. Bock.

Bodem, Con. 1336. Aus den Seiten vorbrech. Scheiben. B.

Poellech ofer, Steph. 1383. Rechter Ober- u. Unter-Ort. B.

Polheim v., Georg. 1373. Drei Schragbalken. Oest.

Bolhen v., Alb. 1333. Vorbrech. Hisch. In der Urk. steht: Albero de Wohlen. B. 
Poppenberger, Albert. 1320. Zwei Schrigbalken. B.

- Ulrich. 1313. Rüdenkopf. B.

Poschendorf v., Johann. Mit Spitzen unt. Haupt gelüngt.

Braiteneck r., Werner. 1288. Sechsmal gequ. Im Siegel steht: „de Brun“. B. Pranbach r., Rudolph. 1303. Schrägfluss. Oest.

Pranter, Rüger. 1281. Spitze unt. Haupt.

Brauna u v., Fried. 1283. Oben gespitzt. Balke. Oest.

Brenden v., Hermann. 1336. $\nabla$ Helm: Kürn. F.

Protschrein, Dietrich der. 1332. - Vierfüss. Kasten. - Wappen wie die vom Holz in Schwaben.

B run v- Scharpfenstein, Otto. 1329. Balke darunter Faden. Milt. Arch.

Pucher v. Buclirain, Ulich. 136t. Gequ. oben gel. B.

Burbech r., Seifried. 133j. Auf dem Helm gelingte Kugel. daneben rechts im Feld aufrecht. astig. Stamm. F.

Burga in r., Hartmann. 1338. Ring. S.

Purchstetten r., Albrecht. 131i. Hahn. B.

Putzner, Geore. 1394. Steh. nakt. Frauenzimmer. B.

Chaefringer, Alb. 1535. Käfer. B.

Cha elbl - vitulus - Clrich. 13. Jahrh. $\nabla$ Steig. Kalb. B.

Cha erge 1, Rinker. Landshuter Familie. 13j4. Schrigfuss erklimm. Bär. B.

Chaerling v., Ott. 1328. Steig. Dacks. Tyr.

Chalb, Eberh. 130t. Steh. Kalb. Boeb.

Kalentin r., Srfried. 13ł3. Schridggestelltes Hackmesser. B.

Kamerit, Ottokar der. 13.1. Mit Kapuze bekleid. Wolf. B.

Karlsbach v, Alb. 133j. $\nabla$ Pfabl. - Ist das Wappen der Herant. - F.

Kazenelnbogen, Berta Gräfin v. 1293. O steh. Frau stützt die Linke auf d. Rienecker Schild, hailt in der Rechten d. Helm dieses Geschlechtes. Milt. Arch.

Chegler, Ott. 1385. Spitze. B.

Kesselberg v., Fried. 1249. $\nabla$ gross. Kessel über klein. freien Dreiberg. F.

Kirchberg $v$. Raban. 1263. $\nabla$ Sparr. anf gelängt. Schild. F.

Kirchsteiger. Bernhard. 1367. Zwei schmale Schrägbalken. B.

Lirperg r., Con. 137t. Sparre ohne Schildtheil.

Closener, Alb. 1321. $\nabla$ Schwan. Nach Hund, bsyr. St. B. eine schwarze Uttenschralbe im gold. Feld. B.

Knoll r. Gansheim, Marg. 1347. Gebog. Hirschstange. S.

Koenigshofen v., Heinr. 13ñis. $\nabla$ Helm: off. Flug. F.

Chranichsberg r., V7rich. Helm: Kranich. 1349. Oest.

K reglinger, Pet. B. v. Rothenburg. 1379. Stern ïber Sparre. F.

Chrembs r. Gozo, Richter. 1266. Gel. rechts Läwe, links viermal gequ. Oest.

- v. Rüger, Richter. 1283. Auf Schrägbalken: „COT“. Oest.

Christans r., Otto. 1359. Balke auf gelängt. Schild. Sind die Grossen von Trockan. F.

Kuchel r., Con. 1306. Gestürz. Gabelkreuz, Oest.

Kuchler, Hart. 1385. Helm: wachs. Hirsch. Salzb.

Kuchmejster v. Nortenberg, Guta. 1342. Der rechte Schild sechsmal gel., der linke viermal gequ. 
K i chmeister v. Nortenberg, Katharina, geb. v. Stein. Frau Lup. K. v. N. Der rechte Schild viermal gequ., der linke Haupt.

- Anna, des Lup. K. v. X. Frau. 1376. Recht. Schild: Bockskopf, link. gequ., oben gel.

-- v. Bielriet, Lup. 1379. Dreimal gequ. Helm: wachs. Ziegenbock.

- Lupold. 1321. Schild dreimal gequ. Helm: Pfauwedel.

Kuern von der, Dietrich. 1328. Drei Zinnensparren, off. Flug. B.

Kullingen v., Kuno. Frau Kath. v. Ennershofen. 1372. Frau hält zwei Sillilde, im link. ein Balke. Fragment. S.

Dacha a v., Con. 1397. Maver mit Scharte. B.

Daching v., Ortolph. 1368. geschrägt, unt. Haupt. B.

Talheimer, Ortwin. 1338. Mit Spitzen geschrägt. B.

Tann v., Con. Zwei schräggest. Hirschstangen. F.

Tannberg v., Conr. 1342. Helm: Taube zwischen gestürz. Fisch. Oest.

Tanner, Hans. 1335. Spitze unt. Balke. B.

Ta ur n v., Hermann. 1314. gel. S.

Dege nberg v., Stephan. 1394. Fünfblatt. Staude. v. Gewolf. B.

Degenhart, Hartwig v. Weissenstein. 1377. Behut. bärt. Mannsbïste. B. Ist Degenberg. -

Tettelbach v., Friedr. 1333. Hahnenkopf. F.

Te u fel, Con. 1330. langs gekerbt. B.

*Th angen v., Andreas. 1334. Helm: behntete bürt. Mannsbïste. F.

Tylberich v., Dietr. 1364. Wolf, Helm: wachs. Wolf. Oest.

Tobelheimer, Heinr. 1340. Gequ. oben lauf. Wind.

- Wernhard. 1340. halb. Wind. B.

Toemlinger, Nikl. 1366. Flug mit Zinnenmauer im Sch. B.

* Toerring, Friedr. 1301. Drei Rosen zwischen zwei Fad.-Balken. B.

- Heinrich. 1329. gel., l. gequ. $B$.

Topel v., Wichard. 1324. Zwei Spitzen. Oest:

Toss v., Jungol. 1349 Gequ., oben gev. im 3. Feld. Drei Pfăhle. Sachs.

Trimberg - dapif. de Joh. - 1348. Ganskopf. F.

Truchsen v., Ulrich. 1286. Hint. vier Zinnen vorbrech. Bür. Oest.

Tschetz v., Con. 1327. Schragfluss. In den Urk.: „Con. der Meurer.“ Oest. Duba von der Berkha, Honiko. 1347. Geschrinkte gestünmelte Aeste. Boe.

Tulbi ngen v., Hadamar. 1305. Durchlöch. Kloben. Oest.

Tunz, Walth. 1329. Gequ., oben Pfahl. B.

Durrwangen v., Heinrich. 1348. Viermal gequ. F.

Thurn v. dem, Jakob. Fanfmal gestuckter Sparre. Oest.

Tuschl, Hartwig. 1373. Balke. Helm: off. Flug mit dem Balken.

E benhau sen v., Wernh. 1334. Anfgewachs. Lilie.

E berste in v., Irmg. 1358. Poppos Fran. 0 steh. Frau hält in d. Linken d. Schild mit zwei Pfahlen, stutzt die Rechte auf d. Schild mit d. Rose. Bad.

Everst ein Graf v. 1) Otto, 2) Ludwig, 3) Hermann. 1. u. 3. $\nabla$ gek. Löwe. 2. geraut. Schild. 13. Jahrh. M.

Egmont Graf v., Floris, Herr zu Isselstein u. Martendyk. 1515. Gev. mit M. Sch. 1. 4. Vier Sparren. 2. 3. Gegengezinnt. Balke. M. Sch. Löwe. Zwei Greife als Schildhalt. Helm: Federpyramide. 
Eisenbeutel, Con. der. 1326. Mit z.wei Schrügbalken belegt. off. Flug. Oest. Fisen buch v., Friedr. 1283. Stufe. B.

Ekarts a u v., Ernfried. 1302. Nit drei Raut beleg. Haupt. Oest.

- Cadolt. 1373. Im Sechspass Lïwe. Oest.

Ekke, Con. 1335. Pfahl. F.

Ekelin, s. Goteboldi. 1:22. $\nabla$ Zwei Zackenbalken. Oest.

Ey sau v., Wolfger. 1303. Bequast. viereck. Kissen. F.

Ey storf v., Sigfried. 1303. Balke Oest.

- Georg. 1364. Neun Sterne. 3. 3. 2. 1. Gest.

Fllerbach v. Burk. 1319. Gev. S.

Em ersh of en r., Eckard. 1372. Balke. S.

Engelshofer, Ulrich. 1399. Gel. rechts drei Ringe pfalweise, links warhs. Fingel. - Stammwapp. - B.

Ergoldsbeck v., Bercht. 1330. Helm : m. Fetl. best. Hut. Schild : Veh unt. Haupt.

Ersingen v., Ulrich. 1343. geschrinkte Streitkolben. (Aresinger.) B.

Erpfingen v., Ulrich. 13\%9. Veh. unt. Haupt. B.

Erlbeck v., Ruprecht. 1302. Bärenkopf. B. : .....ts

Erring v., Heinrich. 1343. Ring auf gel. Schild. S.

Ernfels v. Wulfing. Schrügbalke. 1345. Oest.

Esch, Robert Herr. 12i5. $\nabla$ Zehnmal gequ. MOM. SIG. NOV. ROBERT. DAIXE. Milt. Arch.

Eschelbeck v., Ott. 1343. Schrig geraut. Balke. Wapp.-Nussdorf. B

Eschenbeck v. Sifried. 1320. Gespitzt. Schrägbalke. B.

Esel v. Külsheim, Heinr. 1876. Schreit. Esel. F.

Espelbach v., Heinr. 1335. Drei Schrägbalk. F.

Et tenstet ter, Jorg. 1399. Gel. Haupt. Helm: Flug.

Eusenhofen v., Ott. 1298. $\nabla$ Drei Schasfscheeren schräg gestellt. B.

- Otto. 1339. Eine Scheere.

Falchau v., Hans 1334. Balke. - Ist Nothaft v. Fulkenau. - B.

Falken st e in v. Kalhoch. 1326. Helm : mit gezackt. Schrïgbalk. bel. Flug. Oest.

- Kalhoch. 1268. Falke auf Stein. Oest.

Velwen v. Gebh. 1297. Helm: Bockgewaff. B.

Vestenberg v., Alb. 1309. Balke. F.

Veuser v. Betlenkeim, Hans. 1343. Geschrïgt, oben achtmal geschr. Mainz.

* Vetter von der Gilgen, Conrad. 2. 1. Lilie. C. S. Frauenbiste. 1327. Die von Donauwbrth stammend. noch in Oest. als Graf. bluh. Vetter. S.

Feuchtwang v., Hermann. 1343. Aus den Winkeln vorbrech. Lilien.

Viehauser, Con. 1332. Greifenfuss. Helm: Scheibe mit 7 Skeptern best. B.

Viehofen v., Ulrich. 1281. $\nabla$ dessin. Schragbalke in dessin. Felde. Oest.

Legende: C. DNI VLRICI DE ENGE SCHALCHESVELT.

Vilmar v. Ude. 1349. Turnierkrag. auf schrigeviert. Schild. Mainz.

Vischach v., Con. 1331. Abgekehrte schwimm. Fische. B.

Visler, Bernh. 1317. Aufrechte Getreidegarben - spät. brennende Fackeln.

Fla ch, Friedr. der. $1303 \nabla$ drei Zwillingsschragbalken. B.

Fl ach a u er Con. Bürg. v. Reichenfall. 1338 abgesetzt. Pfal. B.

Vlacheneckker, Gotschalk. 1349. Helm: Flug mit Schrägbalke bel. Oest. Flitzinger, Conr. 1308. geschrägt, oben geraut. 
Vogt $v$. Vreudenthal, Bert. 1287. In Feld ein Mann m. Hacke auf der Schult. F.

- v. Gansheim. Con. 1316. Spitze in gel. Schilde.

- v. Jubschütz, Thilo. $128 i \nabla$ gek. Löwe.

- v. Stopfenheim, Hillebrand. 1340. Geflüg. schrüge gest. Schaafscheere. F.

Frankenstein v, Indwig u. Siboto, 1. Gek. Löwe. 2. Lindenbaum zwisch.

2 Schililen, rechts achtstr. Stern, links Löwe.

- Iud. v. 132: $\nabla$ Gek. Iöwe.

* Freu de n berg v., Albert, 1369. gequert.

Froeschl, Heinr. Richt. zn Reichenhall. 1345. Frosch. B.

F ürstenberg, Heinr. Gf. 1276. Reit. Sieg. Im Armschild d. Stammwappen. Auf d. Decke zwei Adler. Milt. Arch.

Fulhin, Con. 1338. Schreit. Füllen. S.

Gabelbach, v. Con. 1303. $\nabla$ Geschrïnte Geer. S.

Gae unpeck, Otto. 1332. Gequ. unt. dreireih. Veh. B.

Gannk ofer, Rup. 1330. Veh. unt. Haupt. B.

Gansa, Joh. Stadtricht. zu Reichenhall. 1320. Gans. B.

Gebersdorfer, Con. Schachschrigbalke. 1389. B.

- Gebsattel v., Heinr. 1313. Steh. Bock. F.

Geggenpeunt v., Heinr. 1303. Geschränkte Beil. v. Watt. v. G. B.

Gemlinger, Alb. 1303. Stufenschrïgbalke B.

Gernbach v., Herm. u. Arnold. 1327. Mit Spitz. gel. B.

(i erwig v. Hattenkeim, Con. 1329. Gebog. Frauenarm. Im Siegel: C. de Hetenkeim. Mainz.

Gew olf zum Degenberg, Hans. 1379. v. Degenberg u. Degenhart.

G f a ell v., Con. Gabelkreuz Helm: mit Fed. besteckt. hoh. Stülphut. Oest. Gly we v., Hans. 1335. Wiederhacke F.

Ginae uttinger, Hans. 1378. Viermal geschrägt.

Gold, Ortl. Bg. v. Passau. 1313. Geschr, unt. geraut. Im Sieg. Ortlieb de Patav. Grafenwerth v., Leb. 1310. Gel, rechts Balke.

Graisbach, Elbt. Gf. v., geb. v. Truhend. 1336. Sitz. Frau hält rechts den Schild mit den drei Hiffhörn. - Neiffen - links den von Truhend. B. Greiff, Ott. 1332. Viermal geschrigt. B.

Greusbach v., Lutold, 1302. $\nabla$ Krebs-Scheere. Oest.

* Griessenbeck, Ulrich der. 1361. Zugekehrte Hühne. B.

G rill, Hans v. Eger. 1333. Bär. Boeh.

Gross v. Meckenhausen; Ulr. 1331. Stufenschrïgbalke. F.

Grueb v., Hartmann. 1378. Eberkopf. B.

Gruen, Con, v. der. Helm: off. Flug mit d. Scbrägbalk. W.-Reitzenstein. F.

Grumbach $\nabla$., Weiprecht. 1326. $\nabla$ Mohr Rosenstengel in d. Recht. haltend.

Gunchinger, Ulrich. 1345. Thurm. B.

Gu ntersdorfer, Luipold. 1368. Zweireihiger Schachbalke. B.

Gutene ck, Rainold. Schneckenschnitt. B.

H a g v., Fried. 1326. Schrägbalke. B.

- Asprian. 1363. Oben mit drei Federbüscheln besteckt. Balke. Oest.

- Ortlieb. 1303. Pelz unt. gel. Haupt.

- Heinrich. 1316. gequ. oben gel.

Hag e n a u v.; Elsb. 0 steh. Frau hält d. gesp. Schild, links i. Feld e. Löwe. Hess. 
Hager, Heinrich. 1359. Zwei Oberorte. Oest.

Haichen bach v., Rüger. 1301. A us Berg aufsteig. Spitze. v. Tanberg. Oest. Haidau v., Alb. 134j. Gel. links viermal gequ. B.

- Con. 1324. Gleiches W.-Helm: mit Federn besteckt Kissen. B.

H a i n beck, Heinrich. 1335. Hahn. B.

Hay ten ch e im v., Gebh. 1336. Halb. Einhorn. B.

Hacken berg v., Otto. 1286. $\nabla$ Hacken. Oest.

Hals, Alhaid Gräfin v. 1347. Balke B.

Han a u, Ulrich Gf. v. Im S.Feld: auf dem Helm wachs. Adler. Milt. Arch. H. Harder, Heinrich. 1335. Gebogener mit Schwert bewaff. Arm. B.

Hargkirchen v., Heinrich. 1327. Schräg gestellte Zange. $B$.

Haslang v., Ott. 1315. Mit Eisenhut gelängt. B.

Hasla u v., Ott. u. Chadolt. 1302. $\nabla$ Wolf. Oest.

Ha us ner, Alb. 1337. Mit vier Spitzen gel. F.

Ha u a n, Heinrich. 1341. Geschrünte Geer. B.

Hauzenberg v. Uedunch. 1. wachs. Mond. B.

- Sighard. Abgekehrte Monde.

Heideck v., Con. 1337. Zweimal gequ. Helm: Straussenkopf mit Hnfeisen im Schnabel. F.

- Sophia. Wittwe des Gottf. v. H., geb. Bunggräin von Nürnberg. Steh.

Frau hält in der Linken einen Vogel. $S$.

Heidend orf v., Berchtold. 1268. $\nabla$ drei schrisg geet. Schwerter. Oest.

Helenstein v., Eberh. 1300. Viermal gequ. S.

Henneberg v. Richza, Grafin. 1335. Sitz. Frau halt die Schilde von H. und Hohenlohe. $F$.

Herberger, Eisenreich. 1328 Mit zwei Pfauwedeln besteckt Hat. B.

Herblgtadt v., Frizo. $1335 \nabla$ mit Spitzen gel. F.

Hersinger, Marchard. 1324. Geschacht. Haupt. B.

Heydeck v., Sib. 1474. Wittwe des G. v. H., geb. Grafin von Ortenb. Gev.

1. 4. zweimal gequ. 2. 3. gegengexinnt. Schragbalke.

Hintberg v., Irmfried. 1237. $\nabla$ Seitenstufengiebel. Im Feld links 7 Billete.

3. 2. 1. 1. gest. Oest.

Hirschberg, Sophie Grifin v. 1302. Sitz. Frau zwisch. d. Schilde v. Oetting. und $H$. $F$.

Hirzauer, Ulrich. 1317. Wappen gleich Waldau. B.

Hoven v., Hein. 1341. Alggek. gebog. Arme vid. die Helmzier der Jarsdörfer.

II of von dem, Alb. Bürger von Regensburg. 1347. Geschragt, oben getautet, unten ein $\mathbf{y}$. $B$.

- Hilbrand. 1468. Schild mit dem Löwen, daneben im Feld zwischen Buffelshörnern sitz. Löwe.

- Albert. 1328. Drei Zinnensparren. 1328.

Hof h i mer, Ruger. 13:50. Freier Stufengiebel. B.

Hohenarter, Stephan. 1324. Zweireihig. Schachbalke. B.

Hoh enberg v., Diet. 1299. $\nabla$ unt. geraut. Haupt. 10 Schindeln 4. 3. 2. 1. F.

- Theodorich. 1353. Drei Rauten auf Haupt. F.

Hohen brunn v., Heinrich. 1339. Sparre unt. Haupt. B.

Hohenhart, Hans, 1365. Zwei Oberorte. F. 
Hohenlohe, Con. Gif. v. 1289. Reit.-S. Im Armschilde und den zwei Decken. schilden die Leoparden. In d. Urk. wird er Con. de Teke genannt, und in einer andern desselben Jahres nennt er sich selbst Con. Nob. de Thekke.

Hohen riet v., Fried. 13000. 2. 1. Ballen. Mainz.

Holens t einer, Zachar. 1387. Stainbockgewaff. B.

Holag u t, Heinrich der. 1343. Gebog. Arm. Boeh.

Holz, Hans aus dem. 1321. Drei Rosen schräg gestellt. - Sind die Mautner von Burghausen. $B$.

Horant v. Sassenfart, Heinrich. 1347. Gel. u. mit spitz. gequ. F.

Horant, Heinrich. 1335. Pfal. F.

Horn begk v., Gebhard. 1320. Gestarzt. Hiffhörner auf gel. Schild. B.

Hornburg v., Hermann. 1326. Viermal gequ. Auf dem Helu Büffelhörner. F. H o y a v., Jakob. 1291. $\nabla$ verschlung. Viereck. Oest.

Huett v. Gabein. 1333. Zelt-Hütte. B.

Hu et te n beck v., Heinrich. 1369. Gegitt. Raute. F.

Hundhoch, Jak. 1349. Ueber Dreiberg jagend. Wind. B.

Jahe n s dorf v., Hartlieb. 1331. Zwei Balken. B.

I du ngs beug v., Leup. 1303. Seitwiirts vorbrech. Mählrader. Oest.

Ilch dorfer, Hans. 1345. Mit Katte bekleid. schreit. Fuchs. B.

Ink of en v., Heinrich. 1332. 2. 1. Platten mit Adler belegt. B.

- Gebolf. Finfblatt. Staude. - Degenberger? -

La dend orf v., Dietr. 1293. gel. Oest.

* Lassherg v. Benesch. 1305. Spitze mit Haupht-Stammwappen. Oest.

La uterbeck, Gerhard. 1354. Veh unt. Haupt. B.

Lemperzh of er, Lud. 1336. Steh. Einhorn. B.

Lengenfeld, Hans. 1316. Schrighalke, Helm: sitz. Hund. B.

Lengen felder, Heinrich. 1335. Geschrigt mit Gegenschräglualke. B.

- Con. 1334. Querbalke aber Schrigbalke. B.

Lengfelder, Ulrich. 1379. Schild u. Helm: Wind. B.

Leubelfing v., Ulrich $u$. Ulrich. 1329. 1. Helm: sitz. Hund, 2. mit Balke belegt. Houpt.

- Ulrich zu Esgmohl, Truchsess in Bayern 1319. Kürn. Ist das Wappen der Truchsess v. E., wie er sich im Siegel auch nennt.

Leutzend orfer, Heinrich. 1339. Ueber Fuss viermal gel. $B$.

Leutzenkirchen v., Simon. 1334. Auf Balke: DAN. B.

Leuzenkirch en v., Eberwin. 1346. Aufrecht. gestummelt. Ast. B.

Lichtenegger, Ulrich. 1390. Gebord. gel. Schild. B.

Lieben berg v., Engelbert. 1327. Gequ., oben wachs. Einhorn. Oest.

Lindorf, Con. Schräggelegte drei Rauten. Oest.

Lobenstein v., Sigfried. 1286. $\nabla$ Viermal gel. Oest.

Lonsdorf v., Ulrich. 1345. Mit Spitz. gel. Oest.

Losen st ein v., Rud. 1360. Panther. Oest.

Luterburg, Otto u. Heyso, Grafen v. 137ó. Lowe. Helm: Sieben Pfaufedern (Erstes Verzeichniss.) Mainz.

Lutzenprunn v., Götz. 1355. gel. Schild, darauf Ring. F. 
I age ns der Schreiber. 1345. Rechts vorbrech. Blatt, Helm: mit Federn besteckt. hob. Hut. B.

Markt, an dem Heinr., Bürg. v. Straubing. 132j. Gel. an den Seiten gezahnt. Im Siegel: S. H. frat. plebani de Struh. v. Waller. B.

Marsbach v., Linhard. 1356. Aus Dreiberg aufsteig. Bock. Oest.

Maiselstein v., Con. 1345. Freier Dreiberg. S.

Marschalk v. Elgen, Heinr. 134t. $\nabla$ Fünfmal mit Veb gequ. - Im Siegel M. v. Donnersberg. - $\mathrm{S}$.

-- v. Oberndorf, Heinr. 1342. Wie M. v. E. S.

- Oberndorf, Sigfrier. Drei Turnierk rägen.

- Becksberg, Heinr. 1327. Vier Turnierkrägen. S.

-- Heinr. Fünfreih. Schach. S.

Maenseer, Thom. 1368. Vier Sparren unt. Haupt.

Mass inger, Ulrich. 1345. Pflugschaar. B.

- Berchtold. 1281. Krone. B. - Die spätere Conthurei nahm diese Krone auch in ihr Wappenschild auf -

Massenhausen v., Alb. 1345. Von zwei Sternen beseit. schräggestellt. Beil. B. Meggenhau sen v., Con. 1331. Viermal gel. unt. Haupt, Helm; Stulphut. F. Meichsa u v., Wernhard. 1344. Einhorn. Dest.

Me nd orf v., Ulrich. 1332. Haupt mit Pfahl darunter. B.

Menkofer, Con. 1326. Greifenfuss. In den meisten Siegeln Bärenpranke. B. Mersdorf v., Heinr. 1347. Viermal gestürtt, gesparrt. B.

Metenfeck v., Gottfried. 1332. Gebandet. Wind. B.

Milin v., Hermann. 1338. Adler. F.

Mirs, Dietrich der. 1334. Aus gestïrz. Hut vorbrech. Hirsch. B.

Mirsenk ofen, Friedr. 1326. Mit Eisenhut gel. B.

Mistelbach v., Perchtold. 1311. $\nabla$ gel. links geraut. Oest.

Morspach v., Ulrich. 1346. Zwei Spitzen auf Balke. F.

- Haug Herr v. 1324. Aus Dreiberg vorbrech. geband. Bracke. Oest.

Mulrieder, Marq. 1345. Halb. Einhorn. B.

Münd linge n v., Hans. 1343. Balke. B.

Muenst er $\nabla$., Heinr. Nobil. $\nabla$ Drei Kugeln. S.

Muckenthaler, Erh. 1418. Spring. Vielfrass, wachs. Löwe hält d. Schild. B.

Murringer, Heinr. 1343. Off. mit Balke belegt. Flug. F.

Muschelrieder, Schweiker. 1397. Fünfblätt. Staude. B. Helm: Búffelshorn. B.

Nan nhof en, Marsch. v. Ott. 1371. Seitensparre. B.

Neidow v., Seifried. 1309. Aufrecht. viereck. mit Quasten verseh. Kissen auf gequ. Schild. Oest.

Neuhaus v., Heinr. Herr. 1317. Hundsrose. Boeh.

Neubauser, Con. 1336. Geschrdnkte Rechen. B.

Nitperg v., Alb. 1320. $\nabla$ Mantelschnitt gel. Boeh.

Nopping v., Heinr. Burggraf von Titmaning. 1239. geschrägt. B.

Nortenberg v., Lupold, kais. Küchenmeister. 1255. $\nabla$ Bchräggest. geflüg. Lyra. Im Siegel: L. Küchenm. v. Rotenburg. F. - Note: Heinrich v. Nordenberg wird 1227 von Ludwig v. Stollberg Sohn sein. Brud. genannt $u$. hat gleich ihm im Schilde eine zweithürmige Burg, vide auch Küchenmeister. 
Sotzenhauser, Con. 1336. Mit Eisenhut gel. B.

Obernburg v., Ruprecht. 1352. Sechsmal mit Spitzen bel. Balke. B.

Oberndorfer, Heinr. 1353. Schräggestellte Fischerhacke. B.

Ode v., Heinr. 13. Jahrh. $\nabla$ Pfahl. Oest.

Oetting v., Heinr. Magist. 1309. Geschrïgt, obeu Hiffuorn, unt. Hirschstange.

- Ist das Wappen Tattenbach. -- B.

Oettingen Graf v., Lud. 1320. Helm: Schirmbrett. s.

Oettling v., Altmann. 1343. Viermal geschrägt. B.

Ort, Heinr. an dem. Bürg. v. Reichenhall. 1293. Oben dreiwal gezackter Balke. vid. Brannau. B.

Ortenburg Gräf. v., Elsb., geb. v. Törring. 1449. Kecht. Schild: Stammwapp. v. Ortenb., link. Schild: Stammwapp. v. Törring. B.

Os theim v., Ulrich u. Herm. 133!!. Einmal aufr., einmal gest. Grabscheit. S. Otakaring v. Gerung. 1308. Geschrägt, oben viermal gegengeschrägt. Oest. Ottmaring v., Heinr. 1342. Drei gegengesturzte Ambasse. B.

- Schweiker. 1339 Helm: zugekehrte Adlerköpfe.

Rab, Heinrich der. 1338. Rabe. Thür.

Radeck v. Gerboch. 1306. Rad. Oest.

Raedwitzer (Rabitzer), Ulrich. 1378. Balke. B. - Der Farbe nach dreimal getheilt. Schild. -

Ramsberger, Heinrich. 1337. Rammel. B.

Ram s d or f er, Wolfg. 1342. Schriggest. Gabel. B.

- Barbara. 1447. Zwei Schilde, rechts mit Zinnen geschragt - Ebran v. Wildenb., - links die Gabel.

- Volker. 1318. Zwei aufrechte Gabeln.

Rapperzeller, Ott. 1340. Gev. Feld, 1. u. 2. gegengeschrägt. B.

Recken rode v., Bert. 1332. Zwei Adlerflägel. F.

Reichen v., Hermann. 131j. Gequ. unt. mit Scheibe bel. sS.

Reichen a u v., Heinrich. 1332. Viermal gequ. F.

Reichershofen v., Seibot. 1330. Drei Sterne auf Schrtubalke. F.

Reifers berger, Gottf. 1337. Zwei Rosen auf Balke - manchmal erscheinen Rader. - B.

Keihensdorfer, Wolf. 1353. Gel. hint. geschacht. Oest.

Reikker, Kasp. 1397. Gel. unt. Haupt. B.

Reintaler, Wilhelm. 1348 Helm: mit Federbart verseh. Büffelhorn.

Rieneck, Ludg. Graf v. u. von Loon-Daren. 1213. Reit.-Siegel. Im Armschild 4. Balken, als C. S. im Schilde ein Löwe. Ada s. Frau. Reit.-S. Die Frau sitzt, den Falken auf der Recbten. Unter dem Pferde lauft ein Wind. C. S. Der Rienecker Schild. Milt. Arch.

Rietsende, Ulrich der. 1324. Balke. S.

Rietzend orf v., Ulrich. 1291. Geraut. Oest.

Rinderbach v., Con. Bäger v. Schwäbisch-Hall. 1379. Imbord, an den Ecken je drei Kugeln. 8 .

Roet en peck, Rudolph. 1334. Gequ., zweimal gel. B.

Rorenstat v., Ulrich. 1327. Geschrigt. B.

Rorer, Alb., Bürg. v. Fger. 1339. Schneckenschnitt. Boeh.

Rosen $\mathrm{b}$ erg v., Elsb. 1336. Zwei Schilde, rechts Rose, links Balke. 
Rosenherg v., Hans. Gev. 1. 4. Zaun, 2. 3. Rose.

- Peter. Fünfblatt. Rose im Fünfpass, ober jedem llatt ein goth. M. Oest.

Ross riet v., Conrad. 1333. Gel. vorn viermal gequ. F.

- Rot v., Con. Herr. 1312. $\nabla$ Gel. rechts Löwe, links Balke. - Sind die noch blühend. von Ulm stammend. Freih. Koth. v. Schreckenstein. - S.

R ota u v., Karl. 1315. Schrägbalke, off. Flug mit nach unt. gek. Schrägbalk. B. Rotten burg v., Seifried. 1328. Zinnenmauer nit zwei Thürmen. Tyr.

Ru dmu u d, Rainolt. 1319. Rádenkopf. B.

Ruegsh ofen v., Conr. 1341. Aus Balke aufwachsende Lilie. F.

Ruesdorfer, Ulrich. 1357. Absteig. gekürt. Pfabl. B.

S a cc, Ulrich. 1338. Schrägbalke, Schirmbrett mit d. Balken. W.-Reitzenstein. F. Salcheim v., Ottokar. 1319. Drei Winde übereinander. B.

Saldorf ve, Con. 1338. Hirschstangen in gel. Schilde. B.

Salern v., Ulrich. Unt. Haupt Stufengiebel. B.

Sal za v., Friedr. 1343. Oben von zwei Rosen, unt. v. Stern beseit. Steinbockhorn, ungewöhnl. gross. $\nabla$ Sieg. Th.

Sch a d, Ulrich. 1343. Krebs. F.

Schalckdorf v. Heidenreich. 1322. Zwei Thürnue auf Dreiberg. B.

Sch a u er, Gerhard. 1330. Viermal geschrägt unt. Haupt. B.

Sch a u e n berg v., Fried. 1311. Vier Balk., Helm: off. Flug m. d. Balk. bel. ()est. Schauen berg v., Fried. 1311. Vier Balk., Helm: off. m. d. Balk. bel. Flug. Oest. Sch a u m berg Graf v., Heinr. 1363. Gel. Helm: geb. zugek. Hiffhörner. Oest. Scheckh, Marchard. 1303. Balke auf gel. Schild. Oest.

Schen ck, Hartlieb. 1366. Die behütete Büste der Degenberger. B.

-. Calhoch v. Falkenstein u. Flsbeth seine Frau nennen ihn Sohn.

Schenk v. Arberg, Fried. 1308. $\nabla$ Steb. Hirsch. F.

- Pymissach, Hans. 1367. Spitze unt. Haupt. - Sind die Sch. v. Neideck und Anzenkirchen. - $B$.

- Töging, Ulr. 1351. Schrägbalke. - Der Farbe nach dreimal geschrägt. -- F. Schilbaz, Albrecht der. 1337. Steh. Reiher. B.

Sch innay v., Friz. 1340. Drei mit den Köpfen ins Gabelkreuz gest. Fische. F. Schlu der, Hein., Richt. zu Wasserburg. 1340. Zwei Rauten. Münch. Geschl. B. Schluttenh of en v., Ulrich. 1372. Gespalt. Pfabl. S.

Schnabel v, Helmsdorf, Martin. 1330. Veh. unt. Haupt. B.

Sch naitbech, Ulrich der. Breze. B.

Schnellh arded orf v., Ulrich. 1343. Balke. B.

Schoenacker v. Karpfham, Simon. Gel., rechts drei Sclırägbalken. - Sind die Rainer v. Rain - B.

Schoe n berg von dem, Alb. Gel. Mantelschnitt. F.

Schowen burg v., Otto. 1346. 3. 2. 1. Schilde. M.

* Schrottenberg v., Otto. 1337. Gel. rechts drei gestlirzte Monde. - Sind die aus Tyrol stammend. noch blühend. Freih. v. Sch. -

Schwanfeld v., Con. 1336. Schwan. F.

Schweningen v., Seifried. 1312. Gürtelschnalle. S.

Sch we p permann, Hartung. 1327. Vehandreaskreuz. B.

- Seifried. 1340. Gelst. Andreaskreuz. Helm: mit Knopf u. Fed. best. Hut. B.

Seemann, Ulrich. 1371. Funfmal geschrigt. $B$. 
Seft-miti s-, Fberh. 13:7. Gekr. halb. Löwe mit durch d. Leib gest. Sclıwert. B.

-Seinshein v., Arnold. 1334. Achtmal gel., Büffelshörner mit je fünf Feder büschien besteckt. F.

Siegenheim v., Fried 1329. $\nabla$ Zwei Gänse hintereinander henschreit. B. Siegershofen v., Leutold. 1347. Panther. B.

Sperrvogel, Con., Büry v. Eger. Vogel an schragg. Speer aufflieg. Boeh. Spiegelberg v., Rüger. 1324. Auf Dreiberg drei langgest. Spiegel. Oest. spielberg v. Hageno. 1344. Lilie auf gel. Schild. Oest.

Stal, Conrad der. 1320. Hut mit zwei Büffelhörnern. B.

Stain auf dem, Lud. 1356. Aus Dreiberg aufwachsende Lilie. B.

- Otto. 1334, $\nabla$ Pfahl. B.

Sttaring v., Ortolf. 13. Jahrh. Zweimal gel, einmal gequ. B.

St a u feneck v., Wilhelm. 1306. Sechsmal gequ. Spitze.

Steckelberg v., Kup. 133j. Gel, rechts Löwe, links drei Balken. F.

Stegner, Hernann. 13ə̄9. Steg über Wellen. B.

Steinberg v., Bercht. Otto Heinrich. 1334. 1. 2. eckiges Kreuz auf Dreiberg. 3. freier Stufengiebel. B.

Ste inelbeck, Heinr. 1335. Halb. gekr. Löwe mit durchgestoss. Schwcrt. B. St einheim v., Conrad. 1343. Henne. B.

Steinkirchen v., Con. 1277. $\nabla$ Gequ., oben viermal gequ. B.

- Con. 1258 . Gequ., oben siebenmal gequ. $B$.

* Steinlinger, Lud. 1336. Hahn auf Berg. $B$.

Sternberg v., Albt. 13. Jahrh. Stör auf Berg. Scchrag. F.

Stettinger, Dietrich. 1377. Mit Zinnen geschrigt. B.

- Dietrich. 1338. Im Schild und auf dem Helm ein Wind. B.

Stolberg v., Ludwig domin. 13. Jahrh. $\nabla$ Zweithürm. Burg. - Er nennt Heinrich v. Nortenberg sein. Brud. Sohn. -

Straubing, Perchtold der Scbreiber v. 1321. $\nabla$ Pfug.

- Seifried der Kastner. Schraggestellt. Schlüssel in gel. S'child. B.

Strass v., Johann. 1303. Drei Rauten nebeneinander. S.

- Conrad. Helm mit zwei Ochsenhörnern.

Stre un v., Ulrich. 1359. Gequ., Helm: Gequ. Flug.

- Alb. 1291. Gequ., oben Balke. S. Alb. VCVLFI DE SCHARCENAWE

- Die Streun schrieb. sich spät. noch von Schwarzenau. Obwohl die in der Urk. erscheinend. drei Streun erklaren, dass sic mit unserm Siegel dieselbe befestig., hangt doch bloss das von Albert an. Oest. Strupperg v., Heinr. 1360. Viermal gel. B.

Stuler, Kasp. 1474. Geschragt, oben Veh, Helm: behüt. Mannsbüste. B.

St u mp f s berg v. Bühcl, Con. 1334. $\nabla$ Panther mit abgekehrt. Vogelköpfen. B. Sturgans, Hans. 133\%. Fisch. B.

Sulz v. Rabno. „Filiast. Lud. dicti de Nortenberch.“ 1255. $\nabla$ Sparre. F.

Sulzbach Graf v., Gebh. 1243. Reit.-Sieg. Im Schilde der Hirsch zwischen

Lilien. Versuch, die Wapp. v. Sulzbach-Castell-Ammerthal mit den

v. Hirschberg zu vereinen. In der Legende nennt er sich v. Hirschberg.

Sulzemoos v., Heinrich. 1305. Halber Wind. 8.

Uetzingen v. Con. $\nabla$ Rose. 14. Jahrh. Oest.

Uffenheim v., Alb. 1261. Burg $\nabla$ sind Nordenberger. F. 
Primbs :

Unholtzing v., Ortlieb. 1325. Geschränkte Blätt. uber dreifachen Bogen. B. Cssel (Vssl), Wilhelm. 1361. linkr's (Oberort. B.

Wabern v., Conrad. 1313. Finhorn. B.

W alda v., Bertold. Gequert, oben Iöwe. S. Bert. ron Weltius. $t$.

Wald v., Otto. 1287. $\nabla$ Mit Zacken sechsmal rechts geschrägt, Cuno mit W ellen sechsmal rechts geschrägt.

Waldeber, Heinr. Mil. $1287 \nabla$ Strauss. F.

Wald e ck v., Emmerich. 1346. Adlertlügel. Mainz.

- Georg. 1378. Löwe. B.

- Ortolph. 1346. Fallend. Adler über Balke. Oest.

- Hadamar. 1332. Schill gleich. Helun: Büffelshörn.

Waldenste in v., Simon. 1362. $\nabla$ vier l'fähle. F.

W aller, Walther. 1316. Dreireihig Schachbalke. S.

- Waltber. 1324. Schrïggestelltes Messer. S.

W altzinger, Ulrich. 1378. Finfblatt. Staude. B.

Wat v. Geggenpeunt. 1313. Geschränkte Beile. v. Geggenpeunt. B.

W eichs v., Hein. 1367. Gelängt. rechts Balke. - Sind die Weichser von Traubling, Winzer und Ramspau. $B$.

Weiltingen v., Lupold Butical. 1265. $\nabla$ Helm mit zwei Pfauwedeln. F.

Weisleizdorf v., Johann. 1349. Helm: federbesteckte Breze. B.

W eiters lorf v., Gottfriell. 1342. Gekrönt. Stiefel. B.

Welchenberg v., Dietrich. 1350. Geschränkte Dietriche.

Welden v. (Weldiv), Ulrich. 1327. Gelingt, rechts 3. Balk. S

W eirden berg v., Hans. 1335. Steinbock. F.

- Rud. Gf. 1307. Fahne in Schild, Bischofsmütze auf dem Helm. Oest.

Wernthel, Ulrich der. 1331. Aufrechter Hecht. Helm: Spornrad auf d. Fisch. B.

W e sterstete $n$ v., Fried. 1318. Gequert, oben gelänt. $S$.

Westheimer, Heinrich. 1343. Sparre. B.

Wi es beck v., Ulrich. 1313. Schrägbalke unter Haupt. Hiessen urspranglich Winkler. Salzburg.

Wilbrechtsrode v. Götz. 1334. $\nabla$ Geschrägt. F.

W i mmer, Heinrich. 1337. Viermal gespart. B.

Winterstein $v$. Neidung. 1369. Schrigbalke.

Winzer v. Rüdiger. 1336. Oben gezinnter Balke. B.

*Winzingerode v., Hans. 1346. schriiggestellte Fischerhacke.

Wittchenstein, Sigd. Gf. v. 13. Jahrh. Reit.-Sieg. in Armschild und auf den zwei Deckenschilden 2 Pfuhle. Hess.

Witmaizzer, Ott. 1343. Pfahl unt. Haupt. B.

Wittolshausen v., Con. 1339. $\nabla$ Pfau. F.

Wolfensdorf $v$, Con. 1308. Wolf. $B$.

Zaenger, Caecilia. 1445. Zwei Schilde rechts geschragt - Ecker v. Eck links Zange im Haupt. B.

Zart, Ludwig. 1341. Geschacht unt. Haupt. B.

Zeschli n, Agnes. 1365. Geschrägt. S.

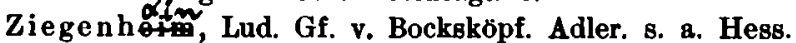

Zolln er, Conrad. 1349. Geband. Rüdenkopf. F. 
Zullenreut v. Ortungk. 1369. Thurm. B.

Zusmegge v., Ulrich. 1301. Rad. Helm: Ochsenhorner. S.

\section{Berichtigungen and Noten.}

Adelzhausen. Auch noch auf einem Siegel von 1340 findet sich der halbe Adler.

Altmannstein. Ulich $v$. Stein hediente sich 1288 des gleichen Wappens. Anderwoldsdorf. Es muss Andermannsdorf heissen.

A ufsess. Das siegel hängt an einer Urkunde von 1326 und nicht von 1322. Aufhausen. - Gebhard v. -

Pappenheim, Marsch. v., Heinrich. Ein Hein. M. v. P. siegelt schon 1256 mit dem gekrönten Mohrenhaupt.

Puchberg. Petres statt Peter.

Castel v. Dietegen. Die von einem zerfallenen Schlosse oberhalb Tagensweiler im Bezirke Kreuzlingen stammenden Constanzer Ministerialen von $\mathbf{C}$ führten in gelängtem Schilde stehenden Leopard. Dietegen bediente sich im Siegel daher nur der Helmzierde.

Villenbach, nicht Villenbeck.

Véllenberg. Muss Villenbach heissen.

Vogel. Das Siegel stammt aus dem Jahre 1523, nicht 1323.

Harbach v, Cuni. Nach einer für das Kloster S. Nikolaus bei Passau ausgestellten Urkunde von 1313 war sie eine Schwester der Grafen Alram und Albert von Hals Sie führt jedoch rechts den geschrïgten Schild und links den mit dem Balken, jene aber den Balkenschild rechts.

Haslach v. Statt Röß̉̀ muss es Ballen heissen.

Henneberg: Die Urkunde ist von 1310, nicht 1300.

Jahensdorf. Die abgekehrten Arme befinden sich im Siegelfelde.

Querfurt. "Gerhard nob. de."

Bampolstetten. Muss Rampoltsheim heissen.

Sept. Pet. der Mayger, nicht Mayper v.

Stau feneck. Das Siegel hat die Umschrift: Wilehalmus capitaneus de Pleygen. Er gehörte aber nach einem Siegel ein. anderen Wilh. v. Staufeneck aus den Jahre 1330 diesem Geschlechte an. 